\title{
An Integrated Agent-Based Architecture and Performance Optimization of VANET using Agent Packet Size Reduction Process
}

\author{
T.Karthikeyan \\ Associate Professor, Department of Computer Science, PSG College of Arts \& Science, Coimbatore, India \\ E-mail:t.karthikeyan.gasc@gmail.com \\ N.Sudha Bhuvaneswari \\ Associate Professor, School of IT \& Science, Dr.G.R Damodaran College of Science, Coimbatore, India \\ E-mail: sudhanarayan03@gmail.com
}

\begin{abstract}
The advancements of Information Technology have led to many developments that make life easier and faster with high reachability and efficiency. There is a drastic improvement in the area of distributed applications with the advent of mobile agent technology over the usual client/server framework. Applying mobile agent technology in the area of distributed applications improves the performance and quality of service. Mobile agent technologies in particular have taken a prominent place in handling effective road and vehicle traffic (VANET). This paper highlights on the integration of previously defined MATLB, PCM and MSA Agent and proposes an approach for reducing the size of the mobile agent that helps in data collection of vehicles in the VANET for effective and efficient traffic control. This paper is an advancement of agent load shedding algorithm and an attempt to optimize the size reduction process. This reduction in size of mobile agents will enhance the performance of VANET making the agents more acceptable by the hosts and correspondingly building an effective co-operative vehicular network.
\end{abstract}

Index Terms - MATLB, PCM, MSA, DoS, RFID, VANET

\section{Introduction}

The Mobile Agent Architecture is a new paradigm applied in distributed networks and this architecture has found a new place recent days in vehicular ad-hoc networks (VANET) also. Mobile agents are programs that are capable of moving from one node to another node in the network carrying vital information and it is a step ahead of the traditional client/server approach [1]. So, Mobile Agents have the flexibility of adapting themselves with any distributed applications [2][3]. In spite of the advantages offered by mobile agents there are few limitations of these agents in terms of number of agents, size of agents and routing time of agents [4]. The performance of this approach depends on two major factors the Cost and Time. An increase in the size of an agent has got a greater impact on these factors. An increase in agent size gradually increases the cost, time and bandwidth utilization.

Background study on the applicability of mobile agents shows how mobile agents can be applied to optimized performance. According to Mostefa and Mohammed mobile agents can be applied on nodes that are grouped together to optimize agent energy requirement and performance of the agent [5]. Shrivatsava Rajesh et.al in their paper have proposed a dummy agent based approach towards building agent security and reduction of overheads by reducing the dummy agent size[6].

Dikaiakos et al [7] proposed a hierarchical framework for the quantitative performance evaluation of mobile-agent middleware platforms. Components of the mobile agent middleware are analyzed using the parameters like operating systems, channel configuration, the size of mobile agent.

This paper proposes a load shedding mechanism using which the size of the agent can be reduced at intervals when agents are employed in carrying information between vehicular nodes. The integrated architecture of agents, where agents are employed in different tasks and the size of the agents can be reduced by programming the control unit and Agent Status Tracker. Reducing the size of agent has a major positive impact on bandwidth utilization, cost and time of packet distribution. The rest of the paper is organized as follows: Section 2 discusses on the application of agents in VANET, Section 3 depicts an agent application scenario, Section 4 gives a detail discussion of Integrated Agent Architecture, Section 5 discusses the Agent packet format, Section 6 about Agent Load Shedding Mechanism, Section 7 about Performance Evaluation and Conclusion and future work are given in the final section. 


\section{Application of Agents in VANET}

A VANET is a form of wireless ad hoc network that aids in establishing communication lines between vehicles and the roadside infrastructures. These VANETs are highly dynamic and needs supportive architecture that aid in efficient management of these networks. VANETs are classified as network on road with dynamic topology, frequent disconnected networks, highly mobile, demands predictions, need to cope up with different environments, demand for sufficient energy and storage, harder delay constraints, interaction with different types of sensors.

Due to the high demanding nature of these roadside VANETs, some additional inputs in the form of hardware or software is required and this support is provided by the mobile agent architecture. Mobile agents are programs with special features developed by Telescript for mobility programming. These agent programs differ from other standard programs in the following aspects: Autonomy, decision making, temporal continuity, goal oriented, mobility, communicative, mutual and learning. The above state features of a mobile agent make it to be more applicable in VANET handling highly dynamic topology, traffic and network.

\section{Agent Application Scenario}

Consider a sample scenario of a road network with mobile agents and this could provide an interesting scenario monitoring environmental changes without any fixed infrastructure. In this scenario the vehicles are equipped with different types of sensors that measure the desired parameter as the vehicles keep moving around inside a particular location that is of interest or the area that is been monitored. The sensors are responsible for data collection and these data collected by sensors are transferred from point-to-point and taken to the information centre or the roadside infrastructure or control room for processing at a later stage. This information from the sensors is carried by the mobile agents that are capable of moving from node to node. The mobile agents take the responsibility of carrying information from vehicle to vehicle on the road network before the message is passed to the control room to keep the vehicles informed about the environment and road conditions. This helps in parallel processing of task monitoring covering a large geographical area without any fixed infrastructure.

For a successful monitoring of the entire network, though a fixed infrastructure is not required, but still the vehicles participating in network need to be equipped with one or more sensors for capturing the data, wireless device for communication purposes and GPS receiver to spot out the location of the vehicles presence and a control unit with system equipped to handle the execution of the mobile agent and co-coordinating the activities of other components in the network [8].

\subsection{Environment Monitoring Process by Mobile Agents}

The process of monitoring the vehicle and the environment to give an updated birds view of the network, the following steps need to be executed.

- Defining the scope of the area that is under consideration with all the necessary parameters to support the process and the period during which the network needs to be monitored and status to be reported are clearly specified.

- The captured data is taken to the control room that is responsible for creating and initializing agents that can move from vehicle to vehicle and is aided with the help of technical devices fit to the vehicles and also the roadside infrastructures.

- During data exchange, it is the work of the mobile agent which can move from vehicle to vehicle and can piggyback itself on the vehicle which has the proximity of travelling towards the area that is under consideration. This assumption of finding the promising vehicle is supported by the GPS information provider and also from the information collected from other nearby agents.

- The entire monitoring process is carried out by the mobile agent by reading the sensors and capability of cloning itself to increase the number of agent instincts in the targeted area to give more accurate monitored data.

- The agent has to stay back in the targeted area for fixed time duration and when the time already defined for monitoring the target runs out, the agent should return back to the control unit with the data or measurement the agent has collected. The process of returning back to the control unit is the same method of jumping from vehicle to vehicle and vehicle to infrastructure.

The above stated mobile agent scenario in VANET shows the advantages of having mobile agents in VANETs in monitoring the road network. Fig. 1 depicts the functionality of mobile agent in VANET.

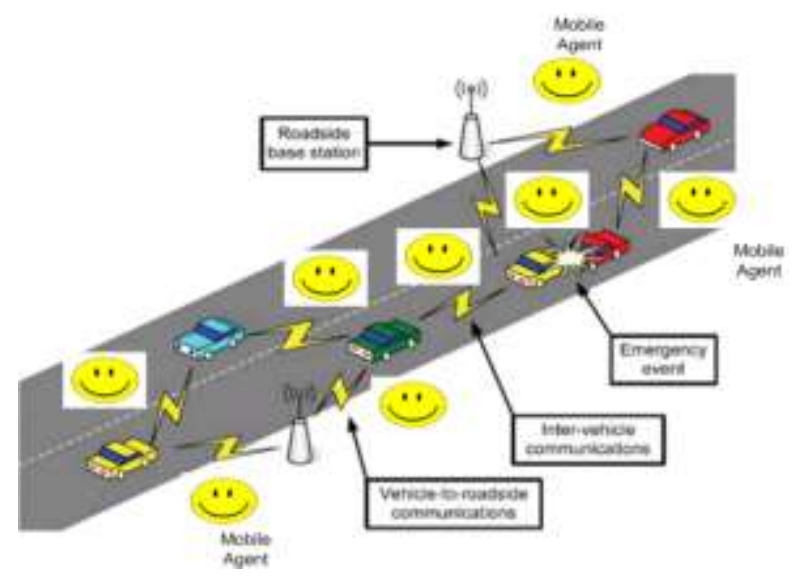

Fig. 1: VANET with mobile agent scenario 
In the above figure, in a road network with bursty traffic, vehicles keep moving to and from in all the lanes. Mobile agents are friendly programs that are capable of reading the input data from the sensors equipped in each and every vehicle on the move. These agents can move from vehicle to vehicle or from vehicle to infrastructure node or the control room and it is also capable of cloning itself to efficiently transfer or carry the messages between various vehicle nodes and the infrastructures.

In the above stated scenario, there is a collision spotted between two moving vehicles and this emergency event has to be notified to other moving vehicles on the same lane, informing the driver to reduce the speed or to take an alternate route, if any is available to avoid the condition of congestion. This message passing has to be done immediately to avoid worsening of emergency situation and this could be very well avoided with the support offered by mobile agents. The emergency situation is sensed by the agent in the targeted area, which clones itself to multiple locations to inform the other vehicles about the current emergency situation, thereby avoiding more collisions or congestions on the onward journey.

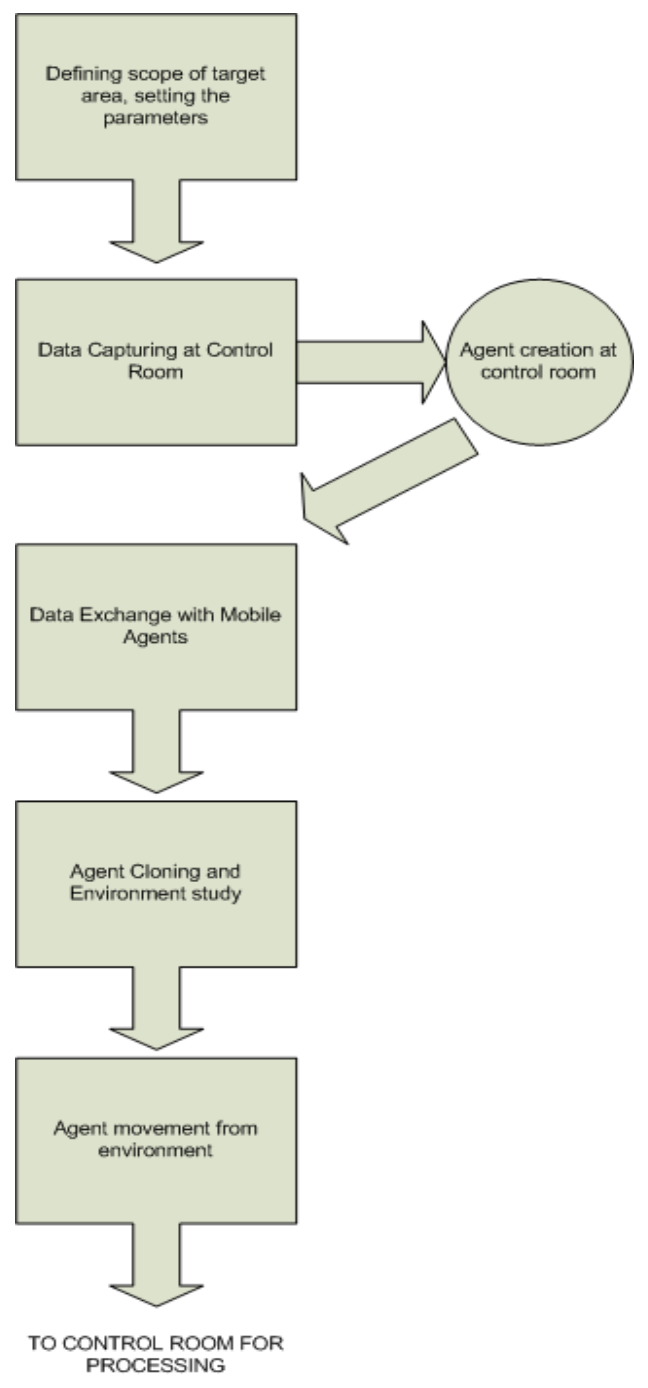

Though mobile agent approach has many advantages there are few limitations of these agents in terms of number of agents, size of agents and routing time of agents. Cost and Time are two major performance factors to be taken in consideration when designing a new architecture. An increase in the size of an agent gradually increases the cost and the time and bandwidth to reach nodes associated with it in the network [9].

This paper proposes a methodology and integrated agent based architecture that integrates many predefined mobile agents like MATLB, PCM and MSA and improves the functionality of the control unit to coordinate these agents and to balance the load of mobile agents that carries the information.

The integrated mobile agent architecture given in Fig 3 refers to already defined agents MATLB. The MATLB (Mobile Agent for Traffic Load Balancing) agent is a conceptual service-oriented architecture based agent supporting interoperability of systems, implementing Ambient Intelligence (AmI) environment.

This agent focuses on building an environment equipped with ambient intelligence that can control the traffic in metropolitan cities without crippling the mobility of users. This agent with sensors, effectors, filters and middleware is one such approach that balances the traffic load and reduces the risks associated with congestion[10].

The PCM agent is an intelligent traffic monitoring agent that controls the speed of the vehicles in a particular lane at a particular location using sensors, mobile agents and RFID technology. The proposed system also categorizes high priority and emergency vehicles to give priority over all the other vehicles in a lane using mobile agent approach making the architecture lightweight. By deploying this agent, vehicles can be controlled at desired locations there by proportionately controlling traffic density and congestion management taking into consideration the movements of real time emergency vehicles[11].

Co-operative intelligent vehicular network is the current proliferation in the transport infrastructure. The potentiality of connected vehicular networks are very high yielding improved passenger safety, congestion control and handling emergency situations. The proposed Multihop Selfless Mobile Agent(MSA) helps in building and maintaining an effective co-operative vehicular network by identifying and isolating misbehaving nodes in the network and also maintaining their credentials and duly discouraging them to act selfishly[12].

The approach proposed here integrates all the above discussed agents MATLB, PCM and MSA in a single architecture and applies size reduction techniques to create light weight mobile agents for efficient performance of agents in VANET.

Fig. 2: Agent Monitoring Process 


\section{Integrated Agent Architecture}

The integrated agents based architecture approach is capable of integrating the functionalities of three agents that forms the backbone for the architecture. The three agents MATLB agent, PCM agent and MSA agent are capable of handling effective network traffic in VANETs. In this integrated architecture the MATLB, PCM and MSA agents should work in co-ordination to achieve the task of handling congestion, giving priorities to emergency vehicles in a busy or congested road and efficient message passing between the infrastructures and vehicles involved in the architecture and identifying selfish behavior of such nodes and helping them to participate in the network to build an effective co-operative vehicular network.

This integrated architecture gives potential solution in case of a VANET with busty traffic and heavy congestion by providing large number of advantages over the traditional client server system approach.

The advantage of this integrated architecture is three fold, where the architecture is capable of handling very high traffic in a busy road that is more prone to congestion and accidents due to very high traffic encountered during regular and peak hours, similarly this architecture has high potentiality of handling emergency situations and giving priorities to vehicles by identifying the vehicles as high priority and emergency vehicles. To enable the above stated emergency situation, it is necessary that the entire road network along with its infrastructure needs to be reliable and this reliability is also achieved by making the network to work co-operatively enabling the nodes to communicate with each other without any interruption or disturbances. All the above said functionalities in a single architecture enable us to build an effective and most efficient road network or VANET.

In Fig 3 all the three agents MATLB, PCM and MSA should work collaboratively. The basic functioning begins with the MATLB agent that takes the responsibility of identifying a congestion scenario in a road and accordingly controls the traffic either by rerouting the vehicles using automated messaging with the help of sensors attached with each vehicular node [9].

The second stage is handling the vehicles in such a way that they do not create congestion on the first hand and creates a risk free traffic environment where emergency vehicles are given priority using PCM agent along with RFID tags and hall effect based sensors that are capable of controlling the speed of the vehicle and creating an accident free zone.

The third stage is the role played by the MSA agent and this agent is capable of communicating with vehicular node and infrastructure node and it can identify misbehaving nodes that are selfish and participate in Denial of Service (DoS) attacks and isolates these selfish nodes are helps in making these nodes to participate in the network activity thereby building a co-operative vehicular network.

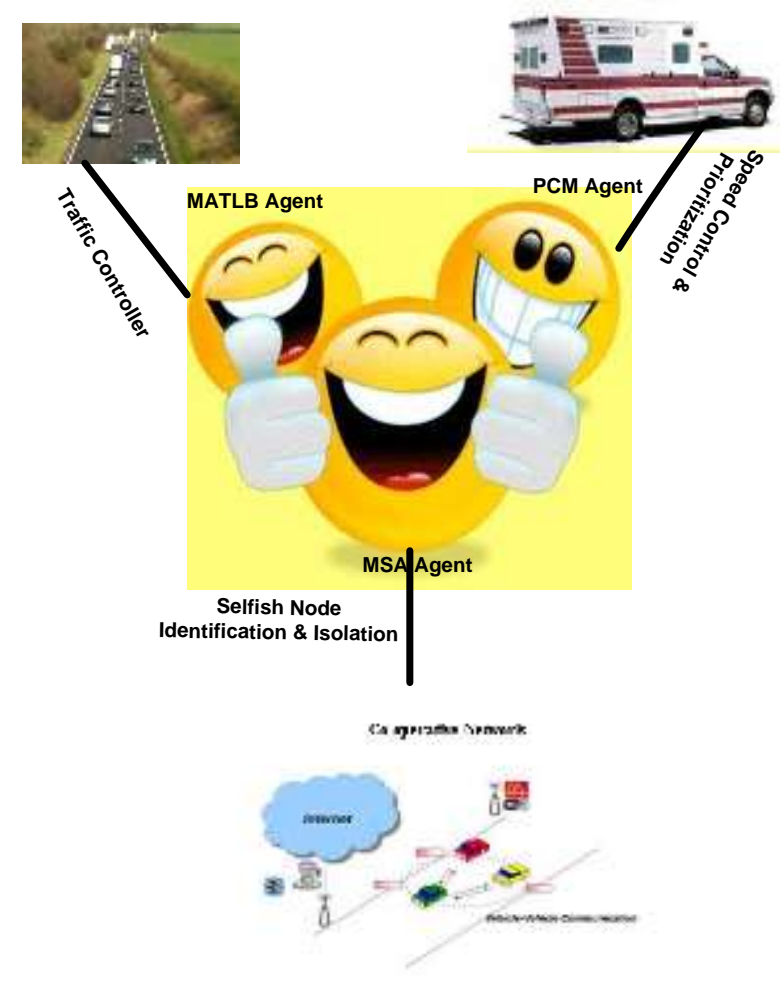

Fig. 3: Integrated Agent Architecture

This integrated architecture takes into consideration the handling of VANET traffic scenario controlling speed, prioritizing vehicle and avoidance of Denial of Service attacks using an agent approach. The next step in this work is towards optimizing the performance of the mobile agent approach that is discussed in detail below.

\section{Agent Packet Format}

Performance is a major factor of measurement in any architecture. In the discussed architecture there are three agents working in co-ordination with each other and one of the important problems that this architecture faces is the size factor of agents. As agents keep moving in the VANET carrying potential information, there is a possibility that their size may increase. This result in some nodes rejecting or refusing the agent due to its size and this also creates problem in increase of time and network traffic and the approach discussed in this paper helps to reduce the size of these agents there by potentially improving the performance of the entire architecture.

Basically the agent basic unit of representation is packets and an agent packet contains information like the type of agent classified as MATLB, PCM and MSA, a unique identifier for each agent, the lifetime of the agent, assigned nodes represent the list of nodes an 
agent has to visit, destination node represents the next node the agent has to visit in its itinerary, the code represents the task to be carried out by the agent and the home node represents the node that is responsible for creating and invoking the agent[13] and the variable component contains variables, their status and their location of usage.

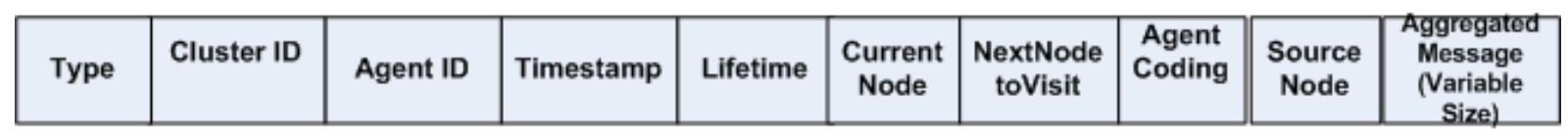

Fig. 4: Agent Packet Format

A normal agent will have the packet contents as stated above. But the problem with agent based approach is the variation in the packet size as the agent moves from node to node that dramatically increases overheads in terms of cost, time and resource utilization. The approach of Agent Load Shedding mechanism is to increase the performance of mobile agents.

\section{Agent Load Shedding Mechanism}

The Agent Load Shedding Mechanism is a process of size reduction of mobile agents for optimization purpose. The various assumptions considered for implementing this mechanism are:

- Agents are mobile.

- They move from node to node to perform tasks.

- Agents require mobility management mechanism.

- Agents are controlled by control units present in distributed locations.

- Control unit takes the complete responsibility of agent size reduction.

In addition to the above made assumptions the various components required for reducing the size of the mobile agent are: Control Unit and Agent Status Tracker. The Control unit consists of other sub components like Garbage Collector, Reconstructor, Data Collector, Security Stamper and Itinerary Changer.

\subsection{Agent Status Tracker}

This agent tracker maintains all important information about the agent maintaining details about the variable, its location of usage and the status of usage of the variable. If the status is set to $\mathrm{ON}$ it is an indication that the agent still needs the variable or element else the usage of that particular element is over.

\subsection{Control Unit}

The control unit works in co-ordination with the subcomponents garbage collector, reconstructor, data collector, security stamper and itinerary changer to help the agent to reduce its size as it moves over the network.
The control unit is the place where the agent can shed its size securely and this unit controls all the other subcomponents. The services to be offered by the control unit are:

- Encrypting and Decrypting of Agent Status Tracker and duly updating the status of this Agent Status Tracker.

- Identifying unused elements and marking them as deleted.

- Rebuilding the agent duly removing deleted elements

- Updating information of new agent in the Agent Status Tracker.

- Making arrangements for the agent to continue its duty.

\subsubsection{Garbage Collector}

This component is responsible for identifying the elements that are to be removed from the agent based on the agent's itinerary and Agent Status Tracker.

\subsubsection{Reconstructor}

With the specification given by the Garbage Collector, Reconstructor takes the responsibility of removing these elements and to rebuild the agent packet for further transmission.

\subsubsection{Data Collector}

This component is responsible for storing the data collected by the agent as on time and prepares a summary of the data collected for future use.

\subsubsection{Itinerary Changer}

This component is responsible for scheduling or rescheduling the itinerary of the agent, if the routing decision of the agent has to be changed dynamically.

\subsubsection{Security Stamper}

This component takes the responsibility of securely moving the agent from place to place using encryption and decryption techniques. 


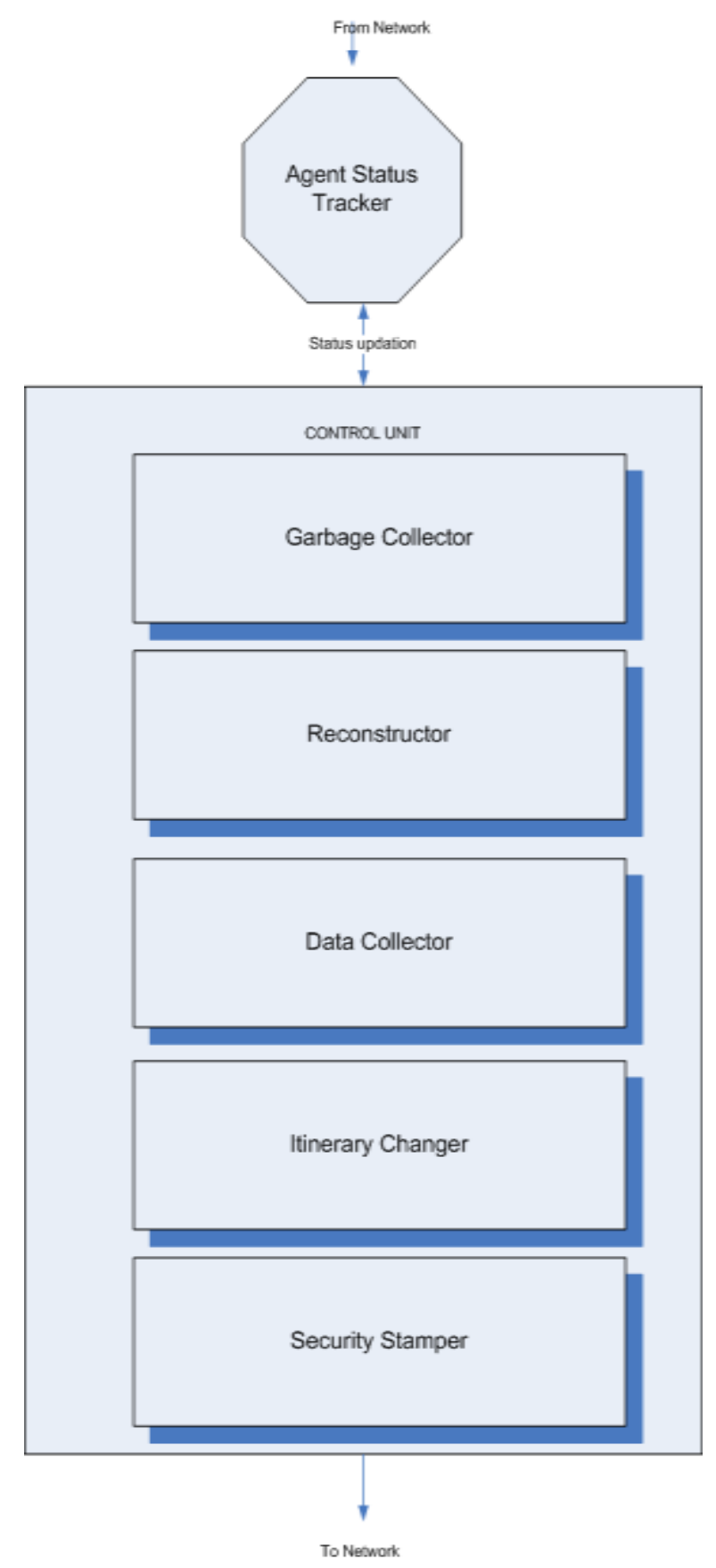

Fig. 5: Agent Load Shedding Components

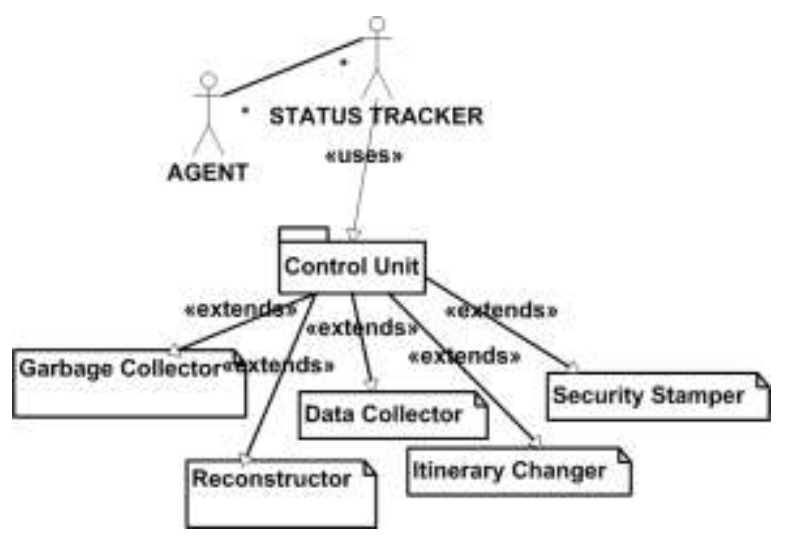

Fig.6 depicts the use case model of the agent load shedding scenario. In this scenario there are two actors, the agents and the agent tracker. The agent tracker keeps track of each and every agent in its domain and when it comes across an agent program that is heavily loaded, the tracker immediately directs the corresponding agent to the control unit. The control unit is a package that has components like Garbage collector, reconstructor, data corrector, itinerary changer and security stamper. All these components work in co-ordination towards reducing the size of agent to maintain an effective network.

\section{Performance Evaluation}

To evaluate the performance of the Agent Load Shedding Mechanism a comparative experimentation was carried out in software developed using .NET framework and compares the agent size reduction before implementing the mechanism and after implementing the mechanism. The results of the experiment are tabulated as given below:

Table 1 : Agent Size before implementing Load Shedding Mechanism

\begin{tabular}{|c|c|}
\hline \multicolumn{2}{|c|}{ Agent Size \& Location } \\
\hline Nodes & Size in Bytes \\
\hline Home Node & 8345 \\
\hline Vehicle Node1 & 8520 \\
\hline Infrastructure Node1 & 8556 \\
\hline Vehicle Node2 & 8623 \\
\hline Vehicle Node3 & 8627 \\
\hline Average & 8534.2 \\
\hline
\end{tabular}

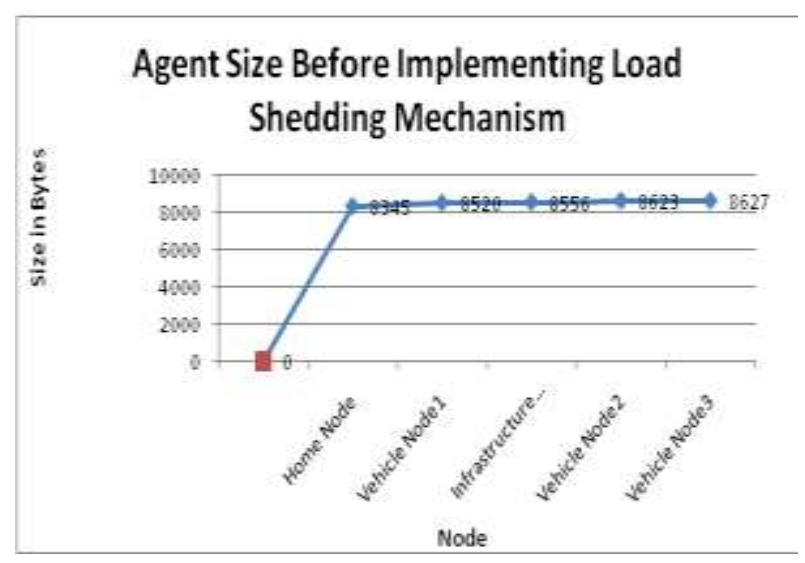

Fig. 7: Agent Size before Load Shedding

Fig. 6: Agent Load Shedding Use Case Diagram 
Table 2: Agent Size after implementing Load Shedding Mechanism

\begin{tabular}{|c|c|}
\hline \multicolumn{2}{|c|}{ Agent Size \& Location } \\
\hline Nodes & Size in Bytes \\
\hline Home Node & 8345 \\
\hline Vehicle Node1 & 8520 \\
\hline Infrastructure Node1 & 8556 \\
\hline Control Unit & 4730 \\
\hline Vehicle Node2 & 4735 \\
\hline Vehicle Node3 & 4780 \\
\hline Average & 6987.2 \\
\hline
\end{tabular}

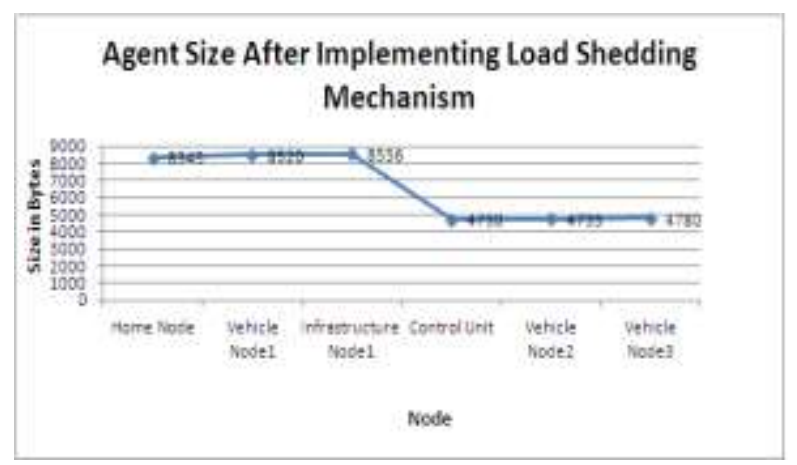

Fig. 8: Agent Size after Load Shedding

\section{Conclusion}

This paper provides an approach to handle the important factor of agent size reduction in an integrated agent's environment using the mechanism of load shedding with the help of the control unit. After applying the mechanism of load shedding there is a dramatic reduction in the size of the agent that is shown in Table 1 and Table 2. This approach is therefore helpful to optimize the performance of the agent based approach to manage the traffic in vehicular ad hoc network effectively and efficiently. The prescribed approach of agent size reduction not only deals with the stated task also but also provides major supportive tasks like managing the road network traffic, congestion detection and avoidance, handling emergency situations and emergency vehicles, selfless service by the nodes i.e either vehicle or roadside infrastructure to provide a seamless uninterrupted service. In future this work can be extended to handle the security issues of mobile agents and the problem of delay in message passing between vehicular nodes and vehicle to infrastructure nodes.

\section{References}

[1] Jin-Wook Baek, Jae-Heung Yeo, and Heon-Young Yeom, Agent Chaining: An Approach to Dynamic
Mobile Agent Planning, School of Computer Science and Engineering, Seoul National University, Seoul, 151-742.

[2] Bo Chen, Harry H. Cheng, "A Review of the Applications of Agent Technology in Traffic and Transportation Systems," IEEE Transaction on Intelligent Transportation Systems, Volume: 11, Issue:2, pages 485 - 497 , 2010-06-00.

[3] Danny B. Lange and Mitsuru Oshima, Programming and Deploying Java TM Mobile Agents with Aglets TM, ISBN 0-201-32582-9, Addison-Wesley, 1998.

[4] J.W. Baek, J.H. Yeo, G. Kim, H.Y. Yeom, Cost effective mobile agent planning for distributed information retrieval, in: Proceedings of the International Conference on Distributed Computing Systems (ICDCS), pp. 65-72. April 2001.

[5] Mostefa Bendjima, Mohamed Feham (2012), Optimal itinerary planning for mobile multiple agents in WSN, international Journal of Advanced Computer Science and Applications, Vol. 3, No. 11.

[6] Shrivastava Rajesh, Mehta,Pooja(2012),Securing Mobile Agent and Reducing Overhead Using Dummy and Monitoring Mobile Agents, International Journal of Managment, IT and Engineering Year : 2012, Volume : 2, Issue : 4.

[7] M. Dikaiakos, M. Kyriakou, and G. Samaras. Performance evaluation of Mobile agent middleware: A hierarchical approach. In Proc. of the Fifth IEEE International Conference on Mobile Agents, LNCS, Atlanta, GA, Springer-Verlag, Dec 2001.

[8] D.Grether, A.Neumann, K.Nagel, 2012, Simulation of Urban Traffic Control: A Queue Model Approach, The 1St International workshop on Agent-based mobility, Traffic and transportation models, Methodologies and Applications.

[9] Michal Pechoucek and David Sislak, 2009, AgentBased Approach to Free-Flight Planning, Control, and Simulation, IEEE Intelligent Systems.

[10] Karthikeyan.T, S.Sujatha, N.Sudha Bhuvaneswari, Mobile Agent Approach for Traffic Load Balancing using Sensors, International Journal of Computer Applications, Volume 47, No. 6, 2012.

[11] T.Karthikeyan, N.Sudha Bhuvaneswari, , S.Sujatha (2012), Traffic Handling Approach with Intelligent Speed Control and Prioritization of Emergency Vehicles using PCM Agent ,International Journal of Computer Technology and Application(IJCTA), Volume 3, Issue 4.

[12] T. Karthikeyan, N.Sudha Bhuvaneswari, An Agent based approach towards building a co-operative 
vehicular network, International Journal of Advanced Research in Computer Engineering and Technology, Vol.6, 2012.

[13] Magdy Saeb, Cherine Fathy, Performance Evaluation of Mobile Agent-based Dynamic Load Balancing Algorithm, Computer Engineering Department, Arab Academy for Science, Technology \& Maritime Transport, 2001.

\section{Authors' Profiles}

Thirunavu Karthikeyan: Thirunavu Karthikeyan received his graduate degree in mathematics form Madras University, Post graduate degree in Applied Mathematics from Bharathidasan University and received doctoral degree in Computer Science form Bharathiar University. Presently he is working as an Associate Professor in Computer Science, PSG College of Arts \& Science, Coimbatore. His research interests include image coding, Mobile agents, Pattern Recognition etc. He has published many papers in National and International Conferences and Journals. He has completed many funded projects with excellent comments.

N.Sudha Bhuvaneswari: Sudha Bhuvaneswari started as a Computer Science graduate. She completed her undergraduate degree at Sri Avinashilingam Deemed University for Women, Coimbatore and completed MCA from Bharathidasan University, Tiruchirappalli and research programme M.Phil in Computer Science from Bharathiar University, Coimbatore, India, and is currently pursuing her doctorate in Computer Science. Her area of interest is Mobile Agent Technology \& Networks. She has been participating continuously in research and development activities for the past twelve years. To her credit, she has presented and published technical papers in International Journals, at International Conferences and International Workshops organized by various international bodies like IEEE, WSEAS, and IEEE Explore, ACM. She has published book on Integrating Service Oriented Architecture and Web Services and also contributed chapters in books like Personal Area Network and published articles and working manuals in agent technology. The author is currently employed as Associate Professor at the Dr. G.R Damodaran College of Science, Coimbatore, India. She is an active member of various technical bodies like ECMA, Internet Society of Kolkata and Chennai and acts as a moderator in various international conferences and journals.
Technology and Computer Science(IJITCS), vol.6, no.2, pp.44-51, 2014. DOI: 10.5815/ijitcs.2014.02.06

How to cite this paper: T.Karthikeyan, N.Sudha Bhuvaneswari,"An Integrated Agent-Based Architecture and Performance Optimization of VANET using Agent Packet Size Reduction Process", International Journal of Information 